http://jmscr.igmpublication.org/home/

ISSN (e)-2347-176x ISSN (p) 2455-0450

crossref DOI: https://dx.doi.org/10.18535/jmscr/v8i4.16

\author{
IGM Publication \\ Journal Of Medical Science And Clinical Research \\ An official Publication of IGM Publication
}

\title{
Microbiological Profile of Vaginal Discharge
}

\author{
Authors
Dr Abhijyoti Maharana ${ }^{1}$, Dr Sulin Kumar Behera ${ }^{2}$, Dr Lal Mohan Nayak ${ }^{3}$, Dr Swetalina Jena ${ }^{4}$ \\ ${ }^{1}$ Junior Resident, Dept. of Microbiology \\ ${ }^{2}$ Associate Professor and HOD, Dept. of Microbiology \\ ${ }^{3}$ Professor and H.O.D Dept. of Obstetrics \& Gynaecology \\ ${ }^{4}$ Assistant Professor Dept of Microbiology
}

Veer Surendra Sai Institute of Medical Sciences and Research (VSSIMSAR), Burla, Sambalpur, ODISHA

\begin{abstract}
Introduction and Objective: Reproductive tract infection and abnormal vaginal discharge affects family life and socioeconomic development with adverse pregnancy outcomes, increase morbidity and mortality with emergence of resistance to antimicrobials. The knowledge of the etiological agents of abnormal vaginal discharge, early diagnosis and selection of empiric antimicrobial therapy will be helpful for better outcomes. The present study was conducted to isolate and identify the pathogens causing abnormal vaginal discharge and antimicrobial susceptibility pattern of their isolates.

Materials and Method: This is a clinico-microbiological study, prospective observational in nature, conducted in the department of microbiology, VSSIMSAR, Burla, western Odisha from November 2017 to October 2019, in collaboration with Department of Obstetrics \& Gynaecology after due approval from Institutional Ethical Committee.
\end{abstract}

Results: The present study was conducted on 110 clinically diagnosed cases of RTI with abnormal vaginal discharge $43.6 \%$ were in age group 26-35years followed by $36.3 \%$ in age group 36-45 years. Abnormal vaginal was higher in rural areas (65.45\%) as compared to urban areas (34.54\%) more prevalent among married women (60\%) and higher among multigravida women (46.36\%). In this study most common isolate was Candida spp. (30.9\%) followed by Staphylococcus aureus (8.1\%), Escherichia coli (5.45\%), Klebsiella spp. (3.63\%), Enterococcus spp. (1.8\%) and Trichosporon asahii (1.81\%).Most of the bacterial isolates were sensitive for vancomycin and Linezolid (100\%) followed by Ampicillin (81.81\%) in case of gram positive organisms and Meropenem(100\%) followed by Amikacin(80\%) in case of gram negative organisms. In antifungal susceptibility testing, most fungal isolates were sensitive to Clotrimazole (76.47\%) followed by Fluconazole (58.82\%).

Conclusion: Vaginal discharge was the most common clinical presentation accounting of RTI cases that too in 26-35 years and in rural females. Vulvovaginal Candidiasis most commonly caused by C. albicansand most fungal isolates were sensitive to clotrimazole.

Keywords: Reproductive tract infection (RTI), Abnormal vaginal discharge, Adverse pregnancy outcomes, Antimicrobial sensitivity pattern.

\section{Introduction}

Reproductive Tract infections are the major public health problem among women especially in developing countries. Abnormal vaginal discharge is a common clinical entity having multiple aetiologies. The prevalence of reproductive tract 
infections (RTI) in different parts of our country vary from $19 \%$ to $71 \% .{ }^{[1]}$ The common etiological agents causing RTI includes Chlamydia trachomatis, Neisseria gonorrhoeae, Trichomonas vaginalis, Gardnerella vaginalis, Candidiasis, Treponema pallidum, HIV, Hepatitis B virus (HBV), Hepatitis C virus (HCV), Herpes simplex virus (HSV) and other bacterial infections. Women with RTI may or may not be symptomatic. They may commonly present with symptoms like abnormal vaginal discharge with foul smell and itching, lower abdominal pain, burning micturition, ulcer in and around genital region, inguinal swelling and dyspareunia. ${ }^{[2]}$ Asymptomatic RTI/STI may function as hidden reservoir contributing to the ongoing epidemic of symptomatic STIs including HIV.

RTI pose a threat to fertility which may result in serious consequences in women such as infertility, ectopic pregnancy, preterm labour, miscarriage, stillbirth, low birth weight baby, pelvic inflammatory disease, cervical cancer, increase susceptibility to opportunistic infection and pregnancy associated complications. Moreover, the presence of STI increases the risk of HIV transmission by 3-4 times among young women. ${ }^{[2]}$ Each year thousand die from sequelae of undiagnosed/untreated RTI which necessitates early diagnosis and treatment. So, it is imperative to study the prevalence, etiologic agents and antimicrobial susceptibility pattern to provide therapeutic modalities and prevent adverse outcome.

\section{Aims and Objectives}

1. To isolate and identify the aetiological agents causing vaginal discharge.

2. To study the antimicrobial susceptibility pattern of isolates.

\section{Material and Methods}

This institutional based prospective study conducted in the department of Microbiology, and in collaboration with department of Obstetrics and Gynaecology VSSIMAR, Burla, Odisha after due approval from Institutional Ethical Committee. Total110 patients with complain of abnormal vaginal discharge visiting Gynaecology OPD were included in study during the period from November 2017 to October 2019. A detailed history of each patient with respect to age, socioeconomic status, marital status, personal hygiene, contraceptive practices, sexual practices and behaviour, health seeking behaviour, past and present history of STI and high-risk behaviour of their partners were recorded in form of a semistructured questionnaires. Written informed consent was taken from the patients prior to sample collection. Menstruating, pregnant, carcinoma cervix, uterine fibroids, uterine prolapsed and on antimicrobial therapy patients were excluded from the study.

\section{Collection of Specimens}

Under aseptic precautions per speculum examination were carried out in lithotomy position in married females. In unmarried women discharge was collected using gloved finger after their consent. Readymade commercially available cotton swab with long rigid swab stick of $22 \mathrm{~cm}$ were used for sample collection. Patient name, ID. No was first properly labelled on the adhesive label followed by collection of three high vaginal swabs from patients according to clinical manifestations. The high vaginal swabs were collected from upper part of vagina, rotated there before withdrawing so that exudates are collected from upper as well as lower vaginal wall. The swabs were transported to microbiological laboratory. First swab was used for wet mount examination under microscope, second swab was used for gram staining \& the third swab was used for culture of micro-organisms. ${ }^{[3]}$ 


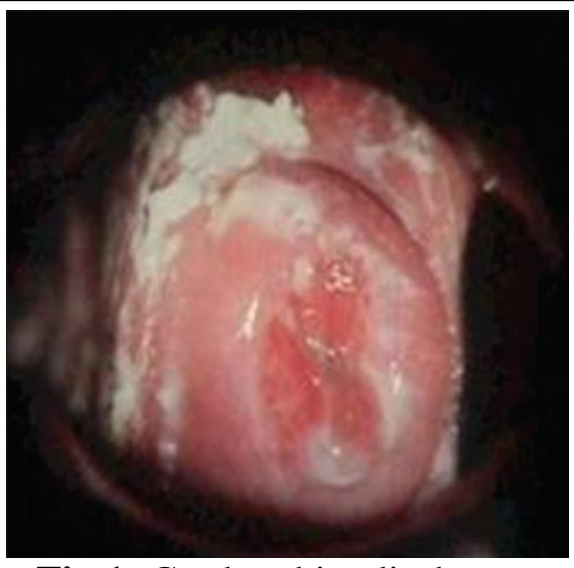

Fig 1: Curdy white discharge

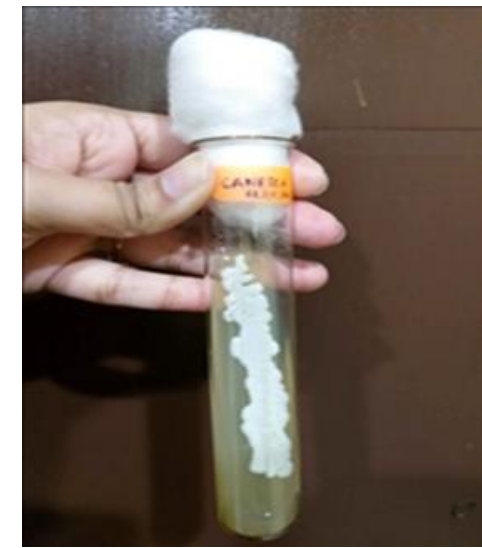

Fig. 2: Growth of Candida species on SDA slant

\section{Transport}

The swabs were transported to microbiology laboratory. ${ }^{[3]}$ Specimen transported at room temperature were processed immediately. In case of delay in processing the specimen were stored at $4^{0} \mathrm{C}$ for up to 48 hours. If culture inoculation is expected to be delay more than 24 hours, then it was stored at -70.C until culture.

\section{Processing of Specimen}

\section{Macroscopic Examination}

a) Observation of sample: Sample was examined for any specific colour and odour. Curdy white discharge was suggestive of candidiasis, greenish frothy discharge was found in trichomoniasis, greyish white foul-smelling discharge in cases of bacterial vaginosis.

b) $\mathrm{pH}$ of vaginal discharge: The $\mathrm{pH}$ of vaginal discharge was recorded using standard $\mathrm{pH}$ indicator paper. Vaginal $\mathrm{pH}$ more than 4.5 was suggestive of bacterial vaginosis.

\section{Microscopic Examination}

a) Wet mount: $\mathrm{A}$ saline wet mount examination was done under low and highpower microscope to detect the presence of motile trophozoites of Trichomonas vaginalis, pus cell and budding yeast cells.

b) Gram's staining: A smear was made from second swab over a clean glass slide and stained using gram's staining technique. The smear was examined in $100 x$ for presence of clue cells, budding yeast cells, polymorphs and Trichomonas vaginalis. Clue cells are squamous epithelial cells covered with small gram variable bacilli.

\section{Culture of Aerobic Bacteria}

The third swab was inoculated into blood agar and MacConkey agar. The plates were incubated aerobically at $37^{0} \mathrm{C}$ for 18 to 48 hours and examined after $24 \mathrm{hr}$ for growth of microorganism and re incubated for another 24 hours in case there is no growth. The organisms were isolated and identified according to cultural characteristic, morphology, gram staining and biochemical reaction as per standard procedure.

\section{Culture of Fungus}

The third swab was also inoculated to pair of Sabouraud's dextrose agar (SDA) media with antibiotics and incubated at $25^{\circ} \mathrm{C}$ and $37^{\circ} \mathrm{C}$ respectively. The isolates were identified based on colony morphology, gram staining, formation of germ tube, ${ }^{[3]}$ chlamydospores formation.

\section{Antimicrobial Susceptibility Testing}

The isolated bacteria were subjected to antibiotic susceptibility testing using Kirby- Baeur disc diffusion method according to CLSI Guidelines. 


\section{Results}

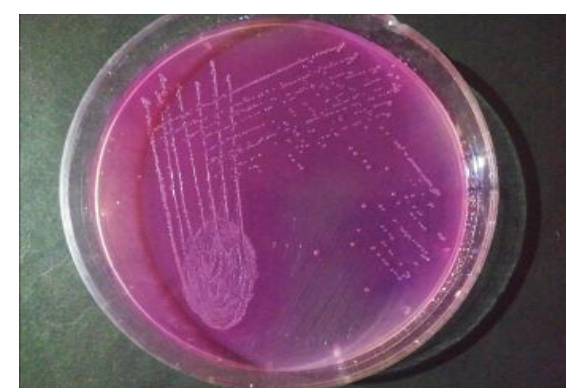

Fig. 3: Growth of E. coli on Maconkeyagar

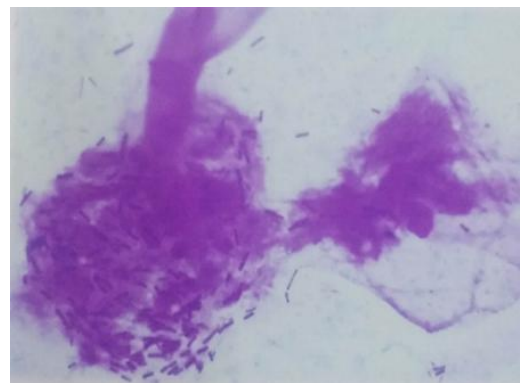

Fig. 4: Predominance of Lactobacilli in Gram stain of healthy vagina

The present study was carried out over a period of 2 years in the Department of Microbiology VSSIMSAR, Burla in collaboration with Department of Obstetrics and Gynaecology. Clinically diagnosed 110 cases of RTI with abnormal vaginal discharge attending OPD of Obstetrics and Gynaecology were included in this study.

Maximum number of clinically diagnosed RTI cases with abnormal vaginal discharge were found in the age group of 26-35 years (43.6\%). Abnormal vaginal discharge was higher in rural areas $(65.45 \%)$ as compared to urban areas (34.54\%) Fig-5. Clinically diagnosed cases of RTI with abnormal vaginal discharge was more prevalent among married women (60\%) than among widow/separated/divorced/unmarried. RTI/ STI increases with increase in parity and was higher among multigravida women $(46.36 \%)$ and those who had undergone medical procedure like abortion $(54.5 \%)$.

In this study, abnormal vaginal discharge was more prevalent among women with low education\& IUCD users. Vaginal discharge was the most common clinical presentation accounting for $(32.7 \%)$ of RTI cases. In this study, RTI cases with abnormal vaginal discharge was most prevalent among IUCD users $43(39.09 \%)$ and least among those using condom 5 (4.54\%) table 1 .

In this study, Gram stain showed Gram positive budding yeast cell $(30.09 \%)$, followed by clue cells $(23.6 \%)$, Gram positive budding yeast cell with altered vaginal flora $(1.81 \%)$, Gram positive cocci (10\%), Gram negative rods $(9.09 \%)$, hyphae with arthrospores $(1.81 \%)$. Wet mount revealed motile trophozoite of Trichomonas vaginalis (3.63\%) by direct microscopy. Fig-6.

In this study most common isolate was Candida spp. (30.9\%) followed by Staphylococcus aureus (8.1\%), Escherichia coli (5.45\%), Klebsiella spp. (3.63\%), Enterococcus spp. (1.8\%) and Trichosporon asahii (1.81\%).

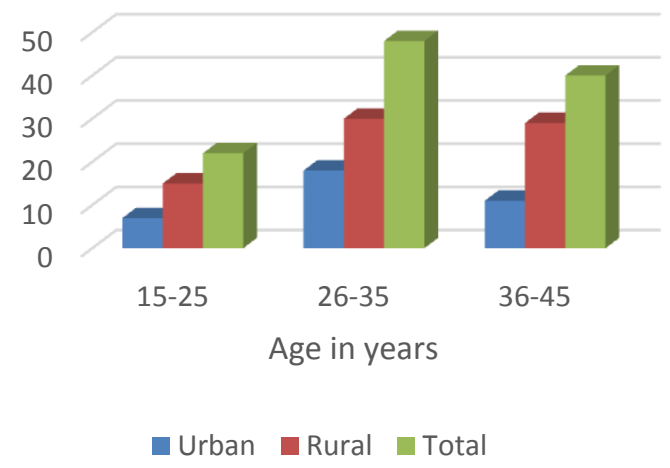

Fig 5 Bar chart showing age group and incidence in rural/ urban population

Most of the bacterial isolates were sensitive for vancomycin and Linezolid (100\%) followed by Ampicillin $(81.81 \%)$ in case of gram-positive organisms and Meropenem (100\%) followed by Amikacin (80\%) in case of gram-negative organisms. In antifungal susceptibility testing, most fungal isolates were sensitive to Clotrimazole $(76.47 \%)$ followed by Fluconazole $(58.82 \%)$. 

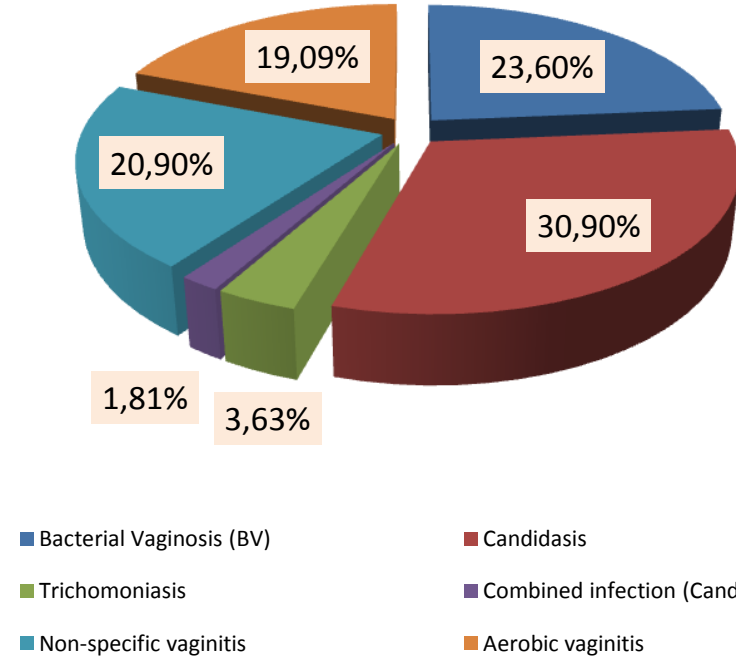

Fig 6 Pie chart showing causating agent of vaginal discharge

\section{Discussion}

Our country is in a state of epidemiological and demographical transition where morbidity and mortality rates vary in different demographic studies. In this study, the prevalence of RTI/STI with abnormal vaginal discharge was highest $(43.6 \%)$ in the age group 26-35 years, because it is a period of maximum sexual and reproductive activity. This finding is in accordance with study conducted by Santra et al. where 56.6\% RTI/STI females were in the age group 20-40 $\mathrm{yr}^{[4]}$

In this study, the prevalence of RTI/STI with abnormal vaginal discharge was found to be higher among women of rural areas (65.4\%) than urban areas (34.5\%).A study conducted in rural district of Agra by Nandan et al. showed the prevalence of RTI among rural women to be $49 \%{ }^{[5]}$ Sarangi L. et al in Sundergarh district of Odisha reported similar prevalence of RTI in rural and urban women. ${ }^{[6]}$ Non-usage of sanitary toilets, use of cloth/homemade napkins during menstruation, poor hygiene, low level of literacy, lack of knowledge of reproductive health among rural women, limited exposure to mass media and interpersonal communication, inaccessibility to health care services due to remote location were the crucial factors which led to higher prevalence of RTIs in rural areas.
Marital status and RTI are related to each other as married women who are leading sexual life have more chance of getting RTIs. In this study clinically diagnosed RTI cases were higher $(60 \%)$ among married women than widow/separated/ divorced/unmarried women. This is in accordance to study conducted by Patnaik L et al. ${ }^{[7]}$ Thus increase in duration of married life leads to increase risk of RTI due to enhanced sexual activity. The prevalence of RTI was low among widow/ separated/ divorced women. Though they are inquisitive about sexual life due to conservative nature of our society, the prevalence of RTI is low in this group.

Education plays a pivotal role in creating awareness among women of reproductive age group. In this study clinically diagnosed cases of RTI with abnormal vaginal discharge were more prevalent among women educated up to Primary school (31.8\%) and illiterate (23.6\%). The prevalence decreases with level of education and RTI was found to be lowest among women with degree followed by those educated up to higher secondary level

In this study RTI was more prevalent among multigravida (46.36\%) than primigravida and nulliparous women. This may be due multigravida being exposed to increased number of deliveries, contraceptive devices and gynaecological surgeries contributing to an increased occurrence of RTI. ${ }^{[8]}$ This study was in accordance to Rathore et al. in which $28.5 \%$ were multigravida, $13 \%$ primigravida and $2.4 \%$ nulliparous women. ${ }^{[9]}$

The current contraceptive status had significant association with prevalence of RTI. In this study the prevalence of RTI was highest among women using copper $\mathrm{T}(43.6 \%)$ and lowest among those using condoms (4.2\%). This is in accordance to study conducted by Patnaik $\mathrm{L}$ et al. where Prevalence of RTI was much higher (40\%) in women using IUCD and no case of RTI was found in barrier contraceptive users. In this study vaginal discharge was the most common presentation $(32.7 \%)$. However most of the patients with vaginal discharge were also associated with 
pruritus, dysuria, low abdominal pain, dyspareunia. This is comparable to study conducted by Nandan et al. In Uttar Pradesh where majority of women $(53.4 \%)$ complained of abnormal vaginal discharge. ${ }^{[10]}$

Most of the cases had curdy white vaginal discharge $(45.4 \%)$ followed by grey colour homogenous discharge with fishy odour (36.36\%). In this study Gram stain shows Gram positive budding yeast cell $(30.9 \%)$ followed by clue cells (23.6\%), gram positive cocci (10\%), gram negative rods $(9.09 \%)$, hyphae with arthrospores $(1.81 \%)$. Wet mount revealed motile trophozoite of Trichomonas vaginalis $(3.63 \%)$ by direct microscopy. This is in accordance to study conducted by Patnaik L et al ${ }^{[7]}$. in Berhamapur city of Odisha where gram stain shows budding yeast cells $(41.3 \%)$, clue cells $(17.4 \%)$ and wet mount showed (15.2\%) cases of motile trichomonas.

In this study candida spp. (30.90\%) were isolated followed by staphylococcus aureus (8.1\%), Escherichia coli (5.45\%), Klebsiella spp. (3.63\%), Enterococcus spp. (1.8\%), Trichosporon asahii $(1.81 \%)$.

Candidiasis was most prevalent vaginal discharge (30.9\%) followed by bacterial vaginosis $(20 \%)$. Our study finding is likeMurugesan et al in which candida spp. (35.42\%) was most common organism identified followed by $E$. coli $(20.8 \%)^{[11]}$. Similar finding was seen in study by Patnaik L et al. ${ }^{[7]}$ in Odisha where candidiasis was most common (41.3\%) cases followed by bacterial vaginosis $(17.4 \%)$.

Most of gram-positive cocci isolated in our study were sensitive to vancomycin and linezolid (100\%) followed by ampicillin (81.81\%). Meropenem (100\%) was the most sensitive drug followed by amikacin (80\%) for all gram-negative bacteria. This is in accordance to study done by Sharma A et al. (2019) ${ }^{[12]}$ at Solapur, Maharashtra. In antifungal susceptibility testing, most of the fungal isolates were sensitive to clotrimazole (76.47\%) followed by fluconazole (58.82\%).

\section{Conclusion}

Young married women in the reproductive age group are more prone to RTI and abnormal vaginal discharge should never be overlooked as it is the most common presenting feature among the sufferers of RTI/STI. Abnormal vaginal discharge further associated with adverse pregnancy outcomes, increase morbidity and mortality. The etiological pattern has been changed in past few years. With emergence of widespread resistance to antimicrobials, the control of infections becomes more challenging. Prevention, early diagnosis and prompt treatment of infections is the rush of the hour. There is a need for creating community awareness and education about health care facilities and instils self-concern in women for their own health needs.

\section{References}

1. Bote MM, Shenoy AG. An epidemiological study to find out the prevalence of RTI/STI and various factors associated with it among ever married women of reproductive age group in an urban slum community of Mumbai. IOSR J Dent Med. 2014;13(3):9-15.

2. Bhattar S, Bhalla P, Rawat D, Tripathi R, Kuar R, Sardana K. Asymptomatic reproductive tract infections /sexually transmitted infections among HIV positive women, Indian J Med Microbiol. 2015;33 (3):410-2.

3. Collee JG, Dugpid P J, Fraser A G, Marimon B P, Simmons A. Laboratory strategy in the diagnosis of infective syndromes. In: Gerald Collee J, Frasser A $G$ eds; Mackie \& Mc Carty Practical medical microbiology .14th ed; Churchil living stone: pp74-75 .

4. Santra S, Lahiri SK. Pattern of reproductive tract infections including sexually transmitted infections among the patients attending a block primary health centre of West Bengal.Int J Community Med Public Health 2015:2(2):149-52. 
5. Nandan D, Mishra SK, Sharma A, Manish J. Estimation of prevalence of RTIs/STIs among women of reproductive age group in District Agra. India J community Med 2002 ;27: 110-3

6. Panda SC, Sarangi L, Bebartta D, Parida S, Panigrahi OP. Prevalence of RTI/STI among women of reproductive age in district Sundergarh (Orissa). Indian J Pract Doctor. 2007; 4:1.

7. Patnaik L,Sahu T,Sahani NC.RTI/STI among reproductive age group in urban slums.Lap Lambert Academic Publ;2012

8. National Guidelines on Prevention, Management and control of reproductive tract infections including Sexually transmitted infections. NACO; Ministry of Health and Family Welfare

9. Rathore M, swami ss, Gupta BL, Sen V, Vyas BL,Bhargav A,et al. Community Based Study of self-Reported morbidity of Reproductive Tract among women of reproductive age in rural areas of Rajasthan. IndianJournal of Community Medicine 2003; 28:117-121.

10. Nandan D, Gupta YP, Krishnan V, Sharma A, Misra SK, Reproductive tract infections in women of reproductive age group in Sitapur/Shahjahanpur District of Uttar Pradesh. Indian J Public Health 2001; 45:8-13 54-16

11. Murugesan M, Armugam V, Gomatheeswari N, Soumya AV . Screening for lower genital tract infections in women og reproductive age group attending a tertiary care hospital. Int $\mathrm{J}$ Repord contracept Obstet Gynecol .2016;5 (11):3987-3992.

12. Sharma A, Rauf SN, Danave DP, Ingole KV. Evaluation of Microbiological Profile and Antibiotic Susceptibility Pattern of Aerobic bacterial isolates in women of reproductive age group with vaginal infections attending tertiary care teaching hospital in Solapur, Maharashtra. Evaluation. 2019 Jan;4(1).145-19. 